\title{
Endobronchial Watanabe Spigot in the treatment of bronchobiliary fistula
}

\author{
Cengiz Özdemir¹, Sinem Nedime Sökücü², Ayşegül Akbaş², Sezin Altay², \\ Ahmet Levent Karasulu', Levent Dalar ${ }^{3}$
}

\author{
1 Yedikule Teaching Hospital for Pulmonary Medicine and Thoracic Surgery, Dept of Interventional Pulmonology, \\ istanbul \\ 2 Yedikule Teaching Hospital for Pulmonary Medicine and Thoracic Surgery, Dept of Pulmonary Medicine, \\ istanbul \\ 3 Istanbul Bilim University, School of Medicine, Department of Pulmonary Medicine, Turkey
}

\begin{abstract}
Bronchobiliary fistula (BBF) is a rare condition in which an abnormal communication exists between the bile ducts and the bronchial tree. Malignancy is the most common etiology of BBF, although many others are possible. A 74-year-old male patient with an inoperable Klatskin tumor presented with a complaint of yellow-green sputum and cough; the patient underwent fiber-optic bronchoscopy based on a preliminary diagnosis of bronchobiliary fistula. Using fiber-optic bronchoscope through rigid bronchoscopy, the laterobasal segment of the lower right lung lobe was occluded using three pieces of 5-mm Endobronchial Watanabe Spigot. Bile drainage subsequently ceased. A bronchoscopic approach provides an alternative option for BBF treatment, particularly in patients who choose not to undergo surgery, or for whom surgery is not an option due to their underlying general condition.
\end{abstract}

Corresponding author: Levent Dalar, Istanbul Bilim University, School of Medicine, Department of Pulmonary Medicine, Turkey.

Phone: +90.5052607170. Fax: +90.2122258391

E-mail: leventdalar@gmail.com

Key words: Bronchoscopy; endobronchial; fistula; spigot.

Contributions: the corresponding author certifies that all authors approved the entirety of the submitted material and contributed actively to the study.

Conflict of interests: all authors declare that they have no conflicts of interest.

The English in this document has been checked by at least two professional editors, both native speakers of English. For a certificate, please see: http://www.textcheck.com/certificate/zyxsU0

Received for publication: 27 September 2013

Accepted for publication: 18 June 2014

CC Copyright C. Özdemir et al., 2015

Tipografia PI-ME Editrice, Italy

Monaldi Archives for Chest Disease Pulmonary Series 2015; 81:747

doi: 10.4081/monaldi.2015.747

This article is distributed under the terms of the Creative Commons Attribution Noncommercial License (by-nc 4.0) which permits any noncommercial use, distribution, and reproduction in any medium, provided the original author(s) and source are credited.

\section{Introduction}

Bronchobiliary fistula (BBF) is a rare condition in which an abnormal communication exists between the bile ducts and the bronchial tree. BBF may be congenital, or may appear following trauma, hepatobiliary surgery, hidatic hepatic disease or malignancy [1,2]. Bile expectoration (bilioptysis) is the primary symptom in patients with BBF. Disease history is also important in preliminary diagnosis.

Depending on the underlying cause, treatment methods including abdominal surgery and endoscopic or transhepatic bile drainage are typically used for BBF; however, treatment is difficult and has high rates of mortality and morbidity. The literature also reports a small number of cases in which bronchoscopic methods have been used for treatment. The Endobronchial Watanabe Spigot (EWS) is a silicone tool used for bronchial occlusion in bronchopleural fistula treatment and control of hemoptysis [3-5]. Bronchial occlusion using an EWS has not been reported previously as a method of BBF treatment. We present a case in which we applied bronchial embolization using an EWS for treatment of BBF

\section{Case report}

A 74 years old male patient presented to our hospital with a complaint of yellow-green sputum and cough. Magnetic resonance cholangiopancreatography had been performed after complaints of itching and jaundice 20 months prior to presentation; a solid mass with irregular enhancement was revealed at the junction of the right and left intrahepatic bile ducts, proximal to the main hepatic channel. Cholecystectomy and choleduct exploration were performed after insertion of a percutaneous biliary drainage catheter.

The mass was found to be a poorly differentiated carcinoma containing a signet-ring-cell component on pathology, and the patient was diagnosed with an inoperable Klatskin tumor. The patient underwent fiber-optic bronchoscopy based on a preliminary diagnosis of bronchobiliary fistula, and the percutaneous drainage catheter was removed upon observing clearing of the bile ducts.

Eleven months prior to presentation at our hospital, an abdominal computed tomography (CT) scan for yellow-green sputum and cough revealed multiple liver abscesses neighboring the diaphragm, and percutaneous abscess drainage was applied. After providing efficient bile drainage and with ongoing bile expectoration, the catheter of the patient was removed and the patient was lost to follow-up. No further surgical intervention was planned due to the general condition of the patient. 
Approximately one month prior to being seen at our hospital, the patient presented to his regular medical center with ongoing complaints of yellow-green sputum and cough. He was referred to our hospital for possible palliative intervention. At initial presentation, the patient's general condition was moderate with normal mentation. Blood pressure was 115/65 mmHg, pulse $98 \mathrm{bpm}$, number of respirations 18/min. Laboratory tests revealed WBC $9420 / \mathrm{ml}$, hemoglobin $11.4 \mathrm{~g} / \mathrm{dL}$, prothrombin time $37.5 \mathrm{sec}$, activated partial thromboplastin time (aPTT) $35.3 \mathrm{sec}$ onds, international normalized ratio (INR) 3.5, AST $44 \mathrm{UI} / \mathrm{L}$, ALT $83 \mathrm{UI} / \mathrm{L}$, and total bilirubin $0.71 \mathrm{mg} / \mathrm{dL}$. Thoracic computerized tomography (CT) revealed bronchiectasis and infiltration in the lower right lung lobe; abdominal CT cross sections showed hepatic abscess formation and dilatation of the intrahepatic bile ducts (Figures la and 1b).

Diagnostic fiber-optic bronchoscopy under intravenous general anesthesia was performed based on a preliminary diagnosis of bron- chobiliary fistula. Yellow-colored bile was observed at the tracheal inlet (Figure 2a), and no endobronchial lesions were seen. Bile drainage was seen to originate from the laterobasal segment of the right lower lung lobe (Figure 2b). The segment inlet was occluded with three pieces of 5-mm Endobronchial Watanabe Spigot (Novatech, Cedex, France) by application of a fiber-optic bronchoscope through rigid bronchoscopy (Figure 3a). Bile drainage subsequently ceased. At follow-up, it was reported that one of the spigots had been expectorated by the patient. Fiber-optic bronchoscopy revealed that the laterobasal segment of the right lower lobe was occluded by only two spigots; no bile drainage was observed (Figure 3b). Figure 4 shows the remaining two spigots in right lower lobe on chest X-ray. The patient was referred to the appropriate facility for drainage of hepatic abscesses. No problems with BBF were reported at three months postprocedure.

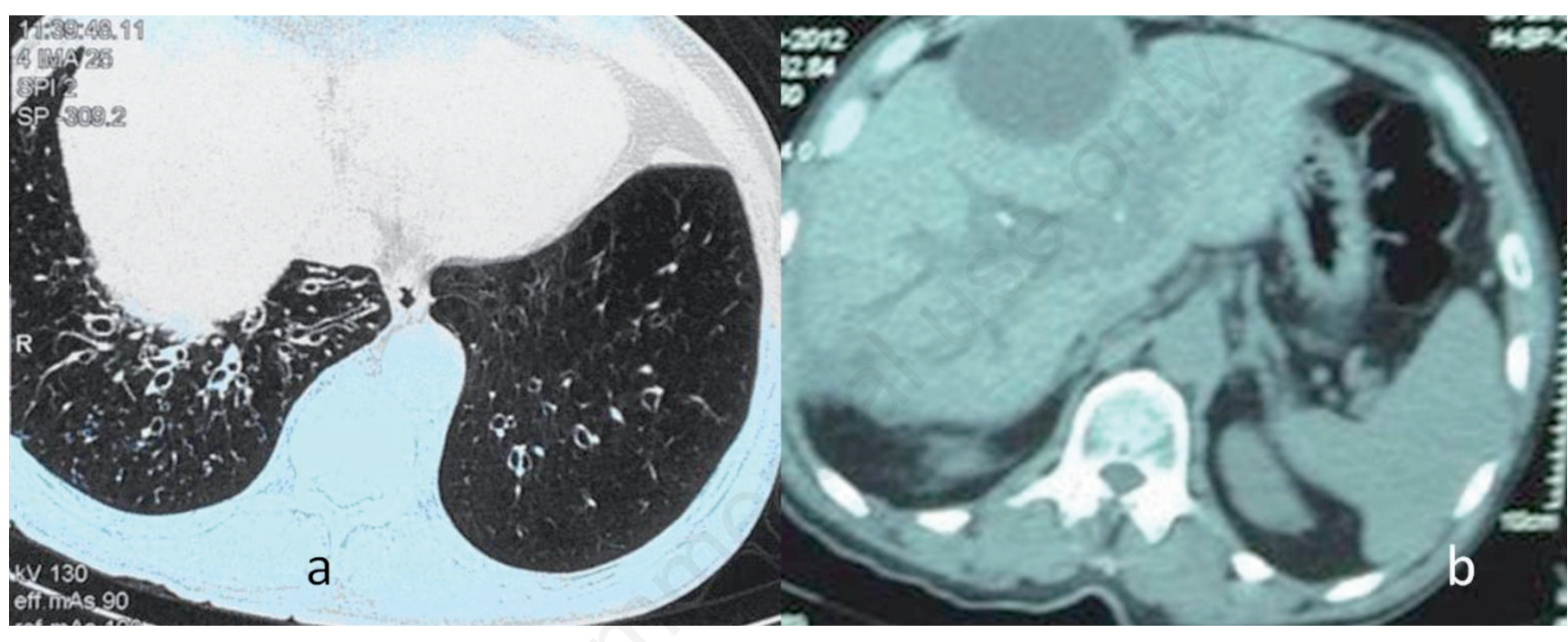

Figure 1. Computed tomography thoracic scan cross sections revealing (a) bronchiectasis in the right lower lobe; in abdominal cross sections (b) formation of abscess in the hepatic anterior, accompanied by dilatation in intrahepatic bile ducts.

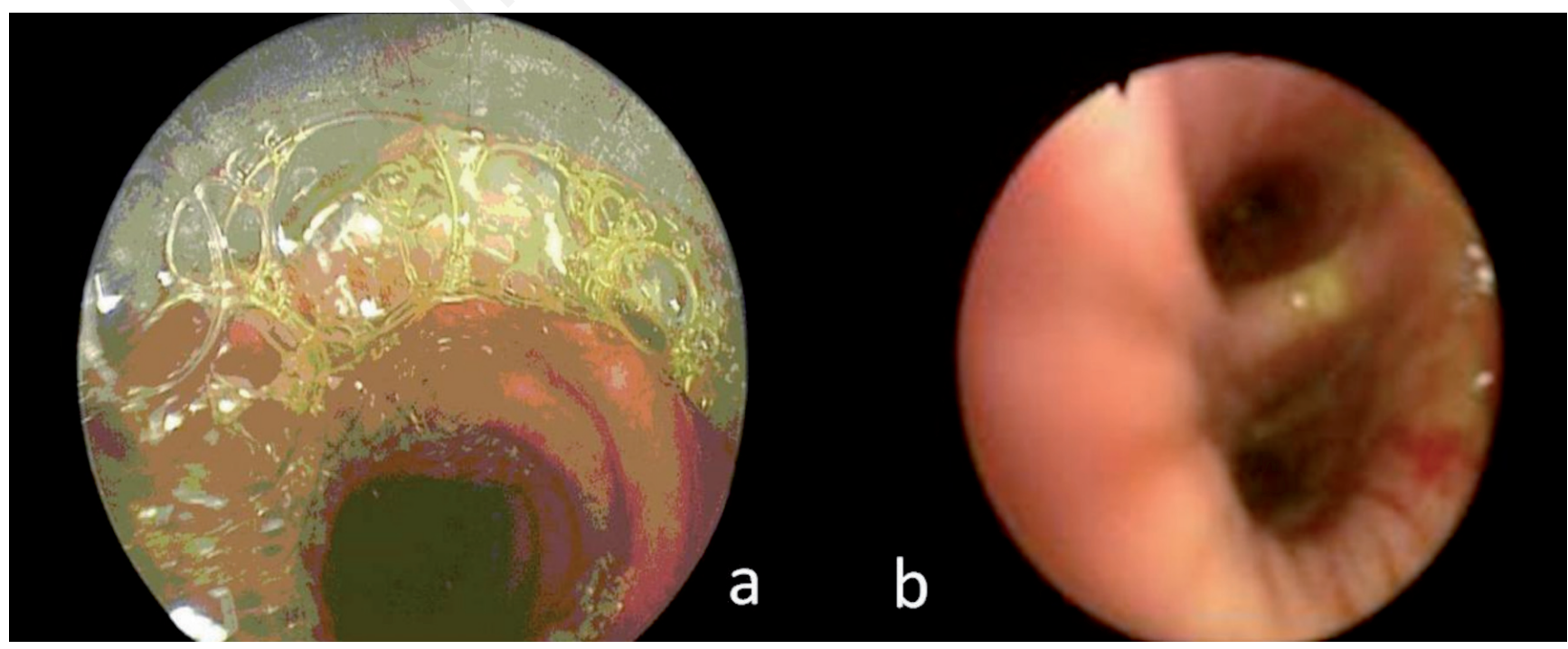

Figure 2. Fiber-optic bronchoscopy performed through rigid bronchoscope shows (a) secretions of yellow colored bile on the mucosal surface after the tracheal inlet (a). The bile drainage originates from the right lower lobe laterobasal segment (b). 


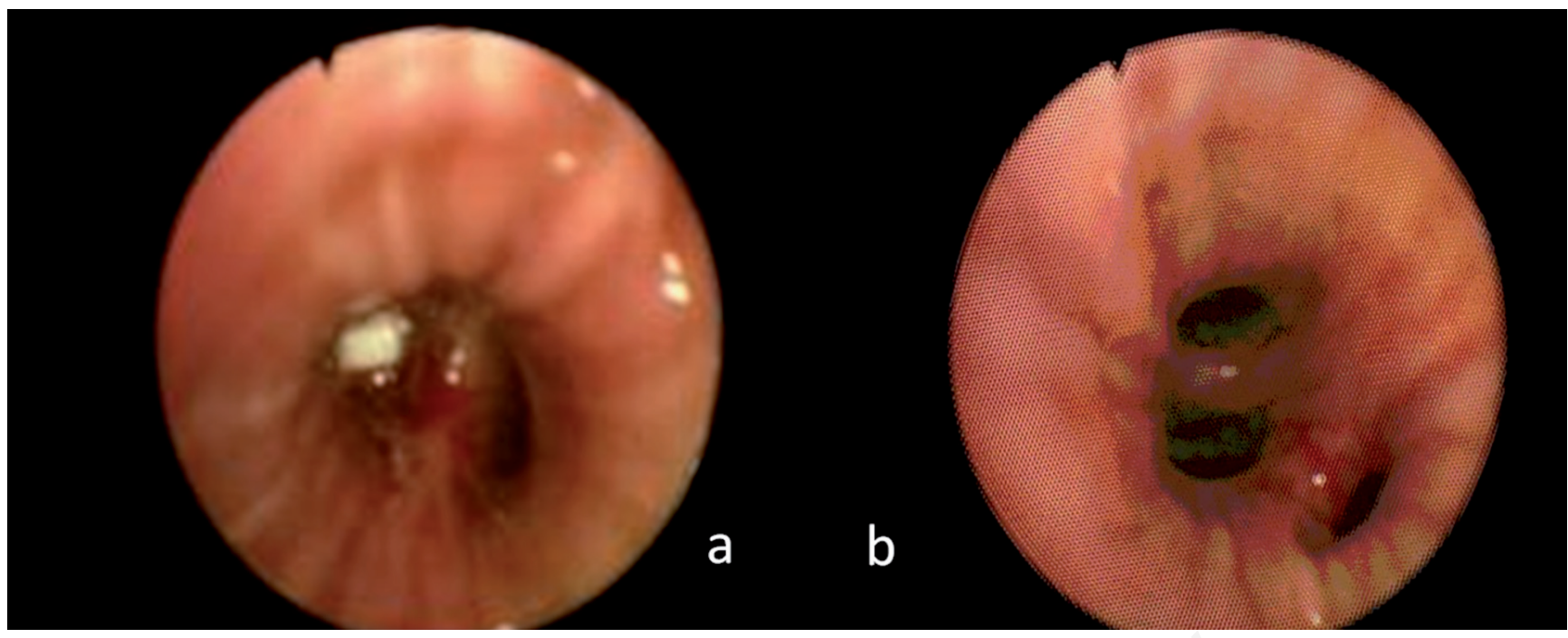

Figure 3. The orifice of the segment was totally occluded by the placed EWS (a) and bile drainage ceased at the level of lower lobe bronchus (b).

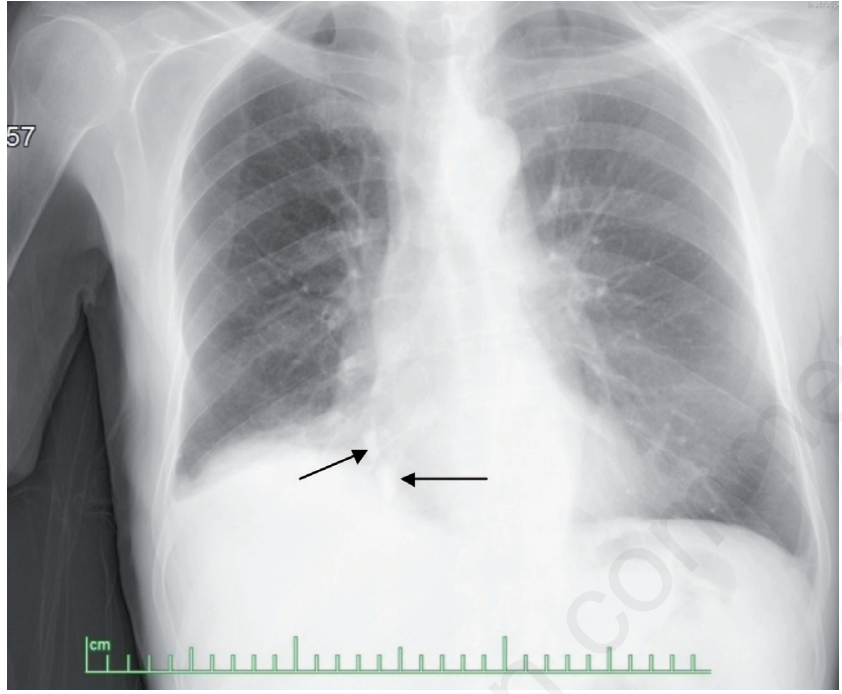

Figure 4. Chest X-ray of the patient after expectoration of one EWS. Remaining two EWSs can be seen in the right lower lobe signed by arrows.

\section{Discussions and conclusions}

Malignancy is the leading cause of BBF, although many other etiologies exist [6]. Various mechanisms play a role in the development of BBF. An abnormal relationship between the bronchial tree and the bile ducts due to bile stones, hidatic cysts, trauma, tumor or postoperative stricture results in BBF development [6,7]. The most important finding in clinical diagnosis is bilioptysis. Yellow-green sputum and cough, radiological findings of accompanying pneumonitis, and underlying disease history are suggestive of BBF [8].

Surgery is the defined conventional therapy for BBF. Methods used include diaphragmatic defect repair with both transthoracic and transabdominal approaches, abscess drainage, pulmonary and hepatic resection, and obstruction of the fistula tract [6]. These surgical proce- dures carry high rates of mortality and morbidity. Conservative approaches may also be used successfully, particularly endoscopic retrograde and percutaneous transhepatic cholangiography [1,9-10]. Although conservative methods were attempted in the case presented here, they were not successful, and no new interventions were considered for the patient due to his general condition.

BBF cases treated with a bronchoscopic approach have been reported in recent years [11-13]. Histoacryl, n-butyl cyanoacrylate, and bronchus occlusion implants made of nickel titanium were used for embolization of fistula tracts in these cases. Patient follow-up and bronchoscopy demonstrated successful treatment of BBF in all cases. After demonstrating in our case that the bile was draining from the laterobasal segment of the right lower lung lobe, the inlet of the segment was successfully occluded with EWS and bile drainage was observed to terminate completely. In previously published cases, bronchoscopic embolization was applied after detection of the fistula tract by an endoscopic approach. In our case, embolization was performed after defining the affected segment by direct bronchoscopic evaluation. The procedure was completed successfully, without imaging of the fistula tract, by observing yellow-colored bile drainage on bronchoscopic examination and correctly localizing the segment of origination.

EWS is a silicone device used mostly for bronchopleural fistula repair and control of hemoptysis. Studies report the successful use of EWS for bronchial occlusion [3,4]. However, BBF treated with EWS has not been reported previously. By occluding the affected bronchus segment with EWS, we ensured termination of drainage to the bronchial tree. Rigid bronchoscopy was used for the sake of airway safety under intravenous general anesthesia, with flexible bronchoscopy performed through it for EWS placement, resulting in a safe, rapid bronchial occlusion procedure.

The most commonly observed complications following EWS application are device migration and atelectasis [4]. In our case, one of the spigots was expectorated $24 \mathrm{~h}$ post-procedure. However, follow-up bronchoscopy demonstrated that the lung lobe segment remained totally occluded, with no bile drainage observed. Secretion retention with associated infection and dyspnea is an uncommon complication following bronchial occlusion with EWS [4]. In our case, bronchiectasis developed in our patient secondary to long-standing BBF. The patient was followed carefully for signs of pulmonary infection in the damaged pulmonary parenchyma following the EWS procedure, and no complications were observed. There is no consensus on the safety of prolonged 
EWS placement in the bronchial tree. In our case, the patient demonstrated no complications during the 3 -month follow-up period, and removal of the EWS devices was not considered.

In conclusion, successful bronchial occlusion with EWS in the case of BBF presented here indicates that it may be used as a new treatment option in these patients. However, a lack of radiological evidence showing the occluded fistula tract prevented conclusive demonstration of success. BBF treatment using a bronchoscopic approach should be considered as an alternative in patients who choose not to undergo surgery, or for whom surgery is not an option due to their underlying general condition or prior treatment failure.

\section{References}

1. D'Altorio RA, McAllister JD, Sestric GB, Cichon PJ. Hepatopulmonary fistula: treatment with biliary metallic endoprosthesis. Am J Gastroenterol 1992;87:784-6.

2. Cropper LD, Gold RE, Robert LK. Bronchobiliary fistula: management with percutaneous catheter drainage of a subphrenic abscess. J Trauma 1982;22:68-70.

3. Watanabe Y, Matsuo K, Tamaoki A, et al. Bronchial occlusion with endobronchial Watanabe spigot. J Bronchol 2003;10:264-7.

4. Sasada S, Tamura K, Chang Y, et al. Clinical evaluation of endoscopic bronchial occlusion with silicone spigots for the management of persistent pulmonary air leaks. Intern Med 2011;50:1169-73.
5. Fujii A, Misumi Y, Hiyama J, et al. Case of intractable hemoptysis controlled by bronchial occlusion with an endobronchial watanabe spigot (EWS). Nihon Kokyuki Gakkai Zasshi 2008;46:416-9.

6. Liao GQ, Wang H, Zhu GY, et al. Management of acquired bronchobiliary fistula: A systematic literature review of 68 cases published in 30 years. World J Gastroenterol 2011;17:3842-9.

7. Pappas SC, Sasaki A, Minuk GY. Bronchobiliary fistula presenting as cough with yellow sputum. N Engl J Med 1982;307:1027.

8. Gugenheim J, Ciardullo M, Traynor 0, Bismuth H. Bronchobiliary fistulas in adults. Ann Surg 1988;207:90-4.

9. Khandelwal M, Inverso N, Conter R, Campbell D. Endoscopic management of a bronchobiliary fistula. J Clin Gastroenterol 1996;23:125-7.

10. Deshmukh H, Prasad S, Patankar T, Patel V. Percutaneous management of a broncho-biliary fistula complicating ruptured amebic liver abscess. Am J Gastroenterol 1999;94:289-90.

11. Kim JH, Kim MD, Lee YK, et al. Bronchobiliary fistula treated with histoacryl embolization under bronchoscopic guidance: A case report. Respiratory Medicine 2008;1:164-8.

12. Goldman SY, Greben CR, Setton A, et al. Bronchobiliary fistula successfully treated with n-butyl cyanoacrylate via a bronchial approach. J Vasc Interv Radiol 2007;18:151-5.

13. Shang Y, Bai C, Yao XP, et al. Transnasal flexible bronchoscopic implantation of a nickel titanium (NiTi) bronchial occlusive device for a bronchobiliary fistula. Endoscopy 2010;42 Suppl 2:E225-6. 